\title{
Le FOU pour ne pas naviguer en eaux troubles
}

\author{
Sebane, Mounia ${ }^{a}$; Tamba, Oumriab
}

aUniversité de Mascara, Laboratoire IPLFS, msebane@yahoo.fr. bUniversité de Mascara, Laboratoire IPLFS, tambaoumria@yahoo.fr

\section{Resumen}

La lengua francesa tiene en Argelia el estatus de lengua extranjera (FLE) en todos los niveles preuniversitarios (primaria, secundaria y universidad). Sin embargo, esta lengua se convierte en lengua de escolarización en la universidad. De hecho, en la enseñanza superior en Argelia, y en particular, en los estudios científicos, como en las ciencias médicas, las ciencias naturales, las ciencias económicas y tecnológicas se imparten exclusivamente en francés. Además de la dificultad para los estudiantes de las asignaturas científicas para construir unos conocimientos científicos en la lengua francesa; también están en la incapacidad de comunicar con el profesor, para asistir a una conferencia o a los trabajos prácticos o dirigidos. Tienen dificultad para leer textos científicos y folletos y por lo tanto no son capaces de tomar notas, sumarios o resúmenes de texto. Este aspecto metodológico es otro obstáculo para la adquisición de contenido científico y la construcción sólida de conocimientos en francés (Sebane, 2008).La demanda cada vez más acuciante para la aplicación del plan de acción de emergencia no sólo proviene de los profesores, sino también de los estudiantes. Por lo tanto, vamos a describir un experimento llevado a cabo como parte de un proyecto de investigación en el CRASC y en el laboratorio de ingeniería de los programas de idiomas en los sectores científicos (IPLFS) para tratar de poner remedio a esta situación y para satisfacer las necesidades de los estudiantes. Este es el montaje de un plan de formación en el propósito del francés para objetivos universitarios (FOU) para los estudiantes matriculados en master 2.

Palabras clave : FOS ; FOU ; conocimientos metodológicos ; las necesidades.

\section{Résumé}

La langue française a le statut de langue étrangère (FLE) dans tous les cycles pré-universitaires (primaire, collège et secondaire) en Algérie. Toutefois, à l'université elle devient langue de scolarisation. En effet, dans l'enseignement supérieur algérien et, en particulier, dans les filières scientifiques, les cours sont dispensés exclusivement en français. En plus de la difficulté pour les étudiants des filières scientifiques arrivés à l'université de construire des connaissances scientifiques solides et en langue française, ils se trouvent également dans l'incapacité de communiquer avec leur professeur, de suivre un cours magistral ou des travaux pratiques ou dirigés. Ils éprouvent des difficultés à lire les textes scientifiques et les polycopiés et sont donc incapables de prendre des notes, de faire des synthèses ou des résumés de textes. Cet aspect méthodologique constitue un autre obstacle à l'acquisition des contenus scientifiques et à la construction de connaissances solides en langue française, (Sebane, 2008). Une demande de plus en plus pressente de mise en place d'un plan d'action en urgence émane non seulement de la part des enseignants mais également des étudiants. Nous allons donc décrire une expérience menée dans le cadre d'un projet au CRASC et au laboratoire d'ingénierie des programmes de langues dans les filières scientifiques (IPLFS) afin de tenter de remédier à cette situation et de répondre aux besoins des étudiants. C'est celle du montage d'un plan de formation en Français sur Objectif Universitaire (FOU) pour les étudiants inscrits en master 2.

Mots-clés : FOS ; FOU ; savoir-faire méthodologique ; besoins.

\footnotetext{
Abstract

The French language has the status of foreign language (FLE) in all pre-university levels (primary, secondary and college) in Algeria. However, it becomes the language of schooling at the university. Indeed, in the Algerian higher education and, in particular, in the scientific fields, such as in the medical sciences, the natural sciences, economics and technology courses are taught exclusively in French. In addition to the
} 
difficulty for students of science arrived at the university to build strong knowledge in French language, they are also unable to communicate with their teacher, to attend a lecture or a practical work. They have difficulty in reading scientific texts and handouts and are there foreunable to take notes, make summaries or text abstract. This methodological aspect is another obstacle in the acquisition of scientific content and the construction of knowledge in French (Sebane, 2008). Demand more and more pressing for implementation of an emergency action plan comes not only from the teachers but also from students. We will there foredescribe an experiment conducted as part of a research project at the research center of social and cultural anthropology (CRASC) and the laboratory of engineering language programs in scientific fields (IPLFS) to try to remedy this situation and to meet the needs of students. This is the assembly of a training plan on French based on Academic Purpose (FOU) for students enrolled in master 2.

Key words : FOS ; FOU ; methodological expertise ; needs.

Notre constat de recherche émane d'une réalité vécue par les étudiants algériens, notamment ceux des filières scientifiques et plus précédemment ceux inscrits en sciences économiques. En effet, ces derniers suivent pendant tout leur cursus scolaire un enseignement scientifique entièrement en langue arabe et une fois à l'université, le français devient et demeure la seule et unique langue d'enseignement dans tous les modules (Sebane, 2011).

Devant une telle situation, les étudiants se trouvent donc dépourvus de bagage linguistique et d'outils méthodologiques ; ce qui entraine des difficultés à suivre le parcours de spécialité en langue française, engendrant ainsi des difficultés de transfert cognitif de la L1 vers la L2 (nous appelons L1 la langue arabe et L2 le français).

L'objectif de notre article étant, de présenter les résultats d'un projet élaboré dans le cadre d'une recherche menée au Centre de Recherche en Anthropologie Sociale et Culturelle d'Oran (CRASC) et le laboratoire de recherche en ingénierie des programmes de l'université de Mascara (IPLFS) qui a pour objectif l'élaboration d'un référentiel de compétences en FOU pour les étudiants de masters d'économie.

Quelques rappels théoriques s'imposent pour tenter de répondre à notre problématique et de poser les hypothèses qui en découleront. Le français sur objectifs spécifiques (FOS) qui est défini comme suit :

Le FOS est l'abréviation de l'expression "Français sur Objectifs Spécifiques”. Il s’agit d'une branche qui fait partie de la didactique du FLE. Ce dernier s’adresse à toute personne voulant apprendre le français dit "général”. Par contre, le FOS est marqué par ses spécificités qui le distinguent du FLE. La principale particularité du FOS est certainement ses publics. Ceux-ci sont souvent des professionnels ou des universitaires qui veulent suivre des cours de français à visée professionnelle ou universitaire (Quotb, 2007 : 68).

C'est une programmation systémique d'un cours de français prenant en compte les objectifs et les besoins d'apprentissage, c'est une approche de la construction du sens dans l'interaction dans une finalité professionnelle ou de formation (non linguistique). En effet, le travail sur le sens dans des contextes universitaires est une condition sine qua none, et non pas sur des structures syntaxiques ou phrastiques isolées dans le but d'assurer une cohérence discursive.

Le FOS comme le souligne Cuq est «né du souci, d'adopter l'enseignement du FLE à des publics souhaitant acquérir ou perfectionner des compétences en français pour une activité professionnelle ou des études supérieures » (2003 : 109).

Le FOS exprime l'usage particulier de la langue, qui porte sur des besoins spécifiques. À Tolas d'ajouter « Leur faire acquérir des techniques de travail destinées à les aider dans leurs activités scientifiques ultérieures : suivre et comprendre des cours, participer à des rencontres professionnelles, lire des articles scientifiques, exposer son savoir et rédiger des travaux de recherche » (2004:07).

En résumé le FOS développe des savoir-faire appropriés à partir d'une analyse de besoins et une connaissance des discours en vigueur. Il met en place un apprentissage de la langue adapté aux besoins des apprenants. En effet, Cuq et Gruca indiquent « Comme son nom l'indique, le français sur objectif spécifique dépend bien entendu de l'analyse des objectifs 
et des besoins. C'est en effet dans cette partie de la didactique des langues que l'analyse des besoins a été le plus utilisée » (2005: 365).

Car les besoins spécifiques sont l'un des principaux fondements de la démarche de la formation du FOS qui consiste à faire acquérir à des étudiants de spécialité des compétences langagières et méthodologiques relatives à leur domaine soit à l'oral et/ou à l'écrit. Car ces publics concernés ne veulent pas apprendre «Le Français mais plutôt Du Français » (Lehmann, 1993 : 115).

Quant au Français sur objectifs universitaires le FOU, il met en avant l'utilité des usages universitaires afin d'atteindre les objectifs d'apprentissage. Autrement dit, les usages et des savoir-faire transversaux tels que la prise de notes à l'écoute d'un cours magistral avec tout ce que cela implique comme investissement tant à l'oral qu'à l'écrit que sur la plan cognitif (Sebane, 2008), la lecture d'un schéma ou d'un tableau, synthèse de documents, résumé d'un cours dans des objectifs bien précis, exposer oralement, la reformulation, l'argumentation, l'explication et la démonstration et l'analyse dans les textes et les discours produits et ou lus, la notion de cohérence, de cohésion, en utilisant les articulateurs logiques ainsi que les anaphores et les progressions thématiques. Tous ces savoir-faire devant être développés et installées en langue étrangère chez les étudiants algériens, ce qui constitue des difficultés supplémentaires.

Face à ce constat, deux interrogations s’imposent : D’où viennent ces difficultés ? Et quelles sont les facteurs qui en sont responsables?

Une problématique en découle :

Comment palier à ces insuffisances linguistiques et méthodologiques pour pouvoir faciliter l'accès au sens des cours et des textes de spécialités ? Est-ce que le FOU en passant par le FOS pourrait être une solution pour combler ces lacunes d’ordre méthodologique car nous supposons qu'une prise en charge d’un besoin méthodologique serait bénéfique pour la construction de connaissances scientifiques solides en L2 ?

A partir de cette problématique découlent des hypothèses :

H1- Le FOS et/ ou le FOU seraient une solution pour combler ces lacunes d'ordre linguistique et méthodologique car nous supposons qu’une prise en charge des deux besoins d’une manière simultanée serait bénéfique pour la construction de connaissances scientifiques solides en L2.

H2- Le manque de corrélation entre le niveau des étudiants et le contenu du cours est le premier facteur responsable de cette difficulté dans la mesure où le niveau attendu est inadéquat au niveau réel.

\section{Expérimentation}

\section{Objectif de l'expérimentation}

Afin de répondre à nos interrogations, nous avons pris comme savoir-faire d'expérimentation la prise de notes à l'écoute du cours magistral de spécialité qui est une situation purement universitaire d’intégration et de construction des savoirs. De plus, elle implique la mise en œuvre des compétences telles que l’écoute, la mémorisation, la compréhension, la sélection d'information et la mise en texte des informations. Elle mobilise des savoir-faire méthodologiques et linguistiques. Enfin, fait important à souligner, cette technique de la prise de notes est inexistante en langue arabe.

De plus, la production écrite reste le moyen le plus adapté à l'analyse et à l'évaluation étant un support palpable, mesurable et quantifiable dans la mesure où il traduit toutes les carences et les performances en langue, c'est une situation d’intégration qui regroupe les savoir-faire et les savoir-être, dans laquelle l'apprenant développe ses compétences. C’est le moyen privilégié à l'évaluation des connaissances en langue et en spécialité. Dès lors nous pouvons insérer l'écriture en activité durant toute la période d’apprentissage pour vérifier la compréhension, la mémorisation et la fixation des notions enseignées ainsi que pour évaluer la maitrise de la langue.

\section{Participants}

Notre expérimentation a touché un enseignant de spécialité et des étudiants de master du département de l'université de Mascara dont le niveau de langue est estimé selon le Cadre Européen commun de références pour les langues entre A1- 
A2 (CECRL, 2000 ; Sebane, 2008) avec lequel nous tentons de mettre en relief toutes les difficultés liées à la compréhension du discours oral à travers notre expérience qui s’est organisée ainsi :

\section{Matériel expérimental}

Nous avons filmé un cours de spécialité en « économie mondiale » et nous avons demandé aux étudiants de prendre des notes des informations importantes du cours et ensuite nous avons analysé les traces écrites afin de détecter les savoirfaire inexistants qui constituent un frein à la compréhension et à la production de texte de spécialités selon la grille suivante élaborée par nos soins pour les besoins de l'expérimentation :

Tableau 1. Grille de critères d'évaluation des prises de notes

\begin{tabular}{ll}
\hline \multicolumn{1}{c}{$\begin{array}{c}\text { Critères observés de } \\
\text { réussite }\end{array}$} & Numéro de la copie \\
\hline Thème repéré \\
Recours à la traduction \\
Correction de la langue \\
Cohérence et cohésion \\
Reformulation personnelle \\
Recours à la synthèse des \\
informations
\end{tabular}

Nous avons donc obtenu les résultats suivants en ce qui concerne les critères que nous avons mis en place :

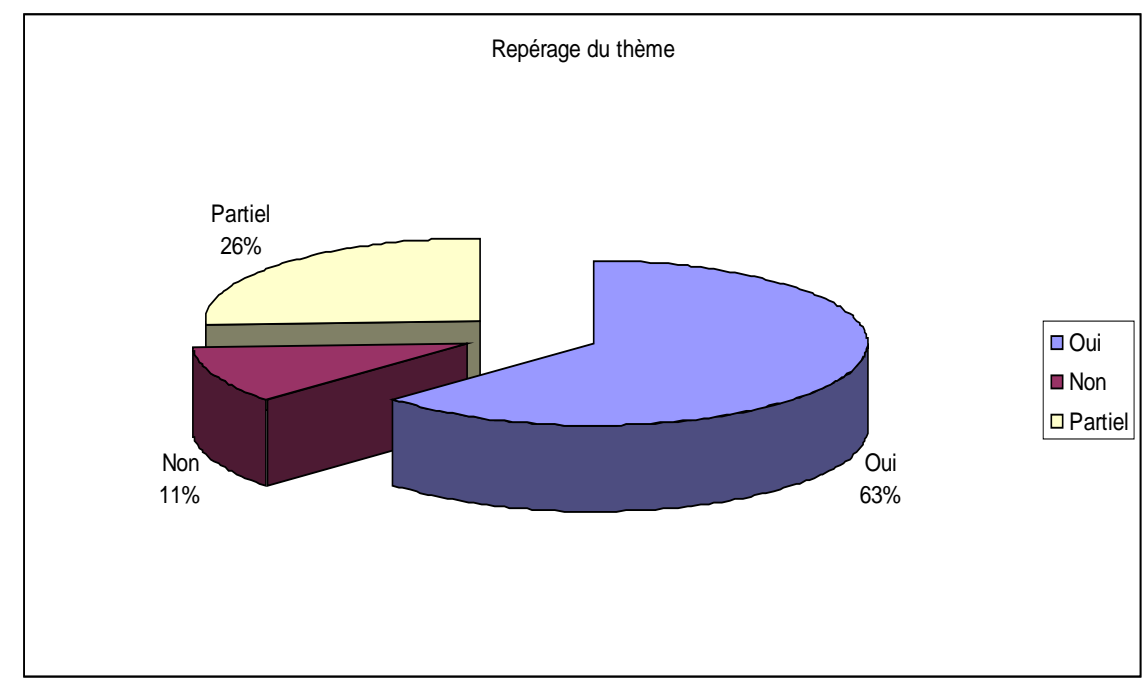

Fig. 1. Repérage du thème

63\% des étudiants ont repéré le thème du cours présenté. $11 \%$ et $26 \%$ entre repérage nul et partiel du thème. 


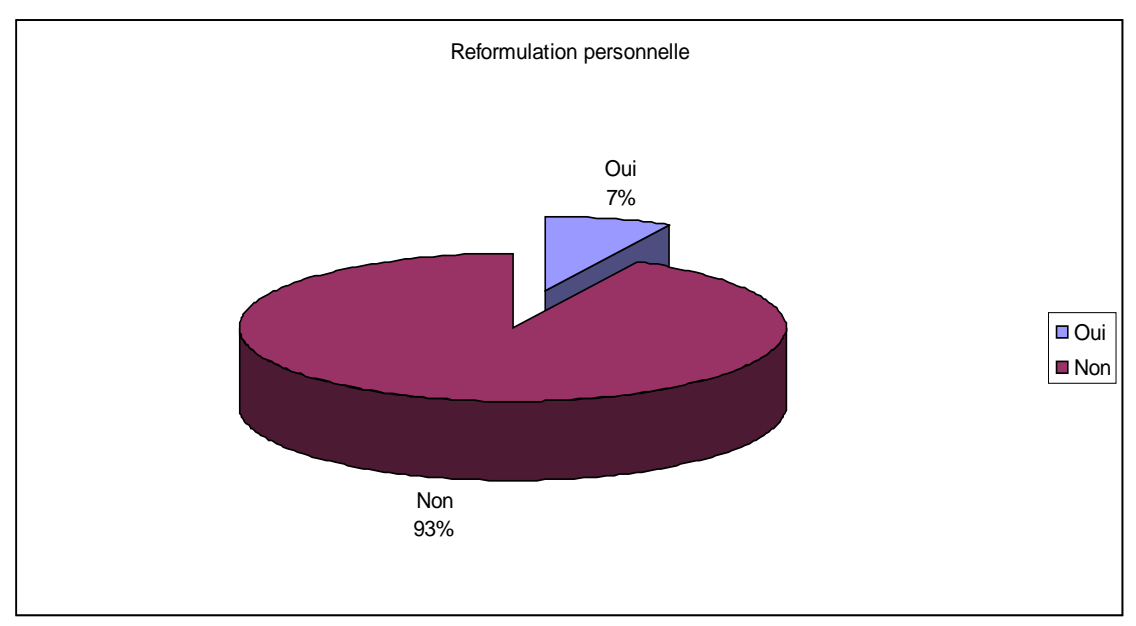

Fig. 2. En ce qui concerne la reformulation

7\% seulement des étudiants reformulent d'une manière personnelle les informations émises par l'enseignant. Dans le reste des copies, des passages entiers du discours de l'enseignant sont copiés mot à mot.

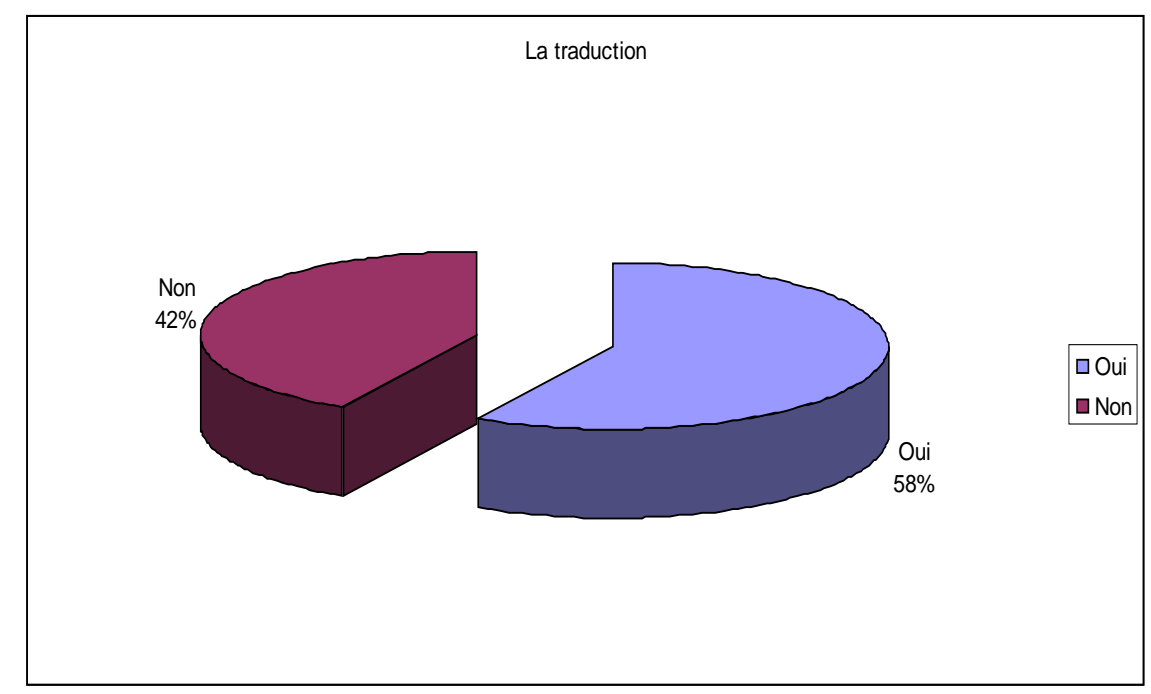

Fig. 3. Recours à la traduction

58\% recourent à la traduction arabe-français. 


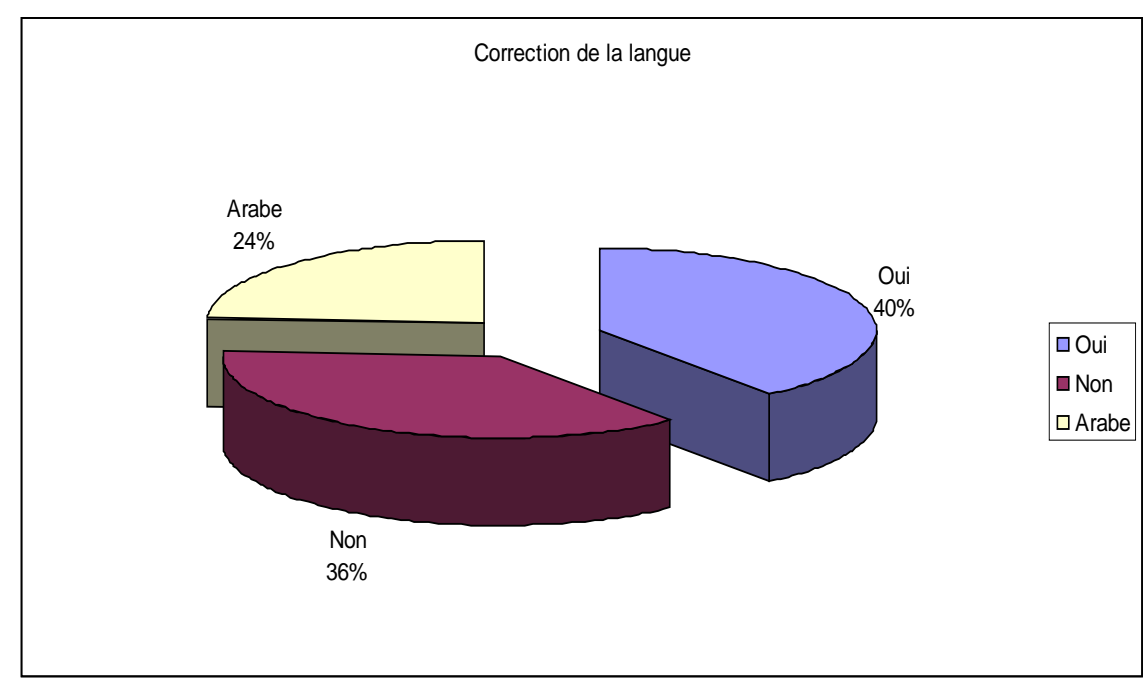

Fig. 4. Niveau de la correction de la langue

Dans $24 \%$ des copies, les notes sont rédigées en langue arabe, 40\% prennent des notes en langue française et $36 \%$ ne prennent pas de notes.

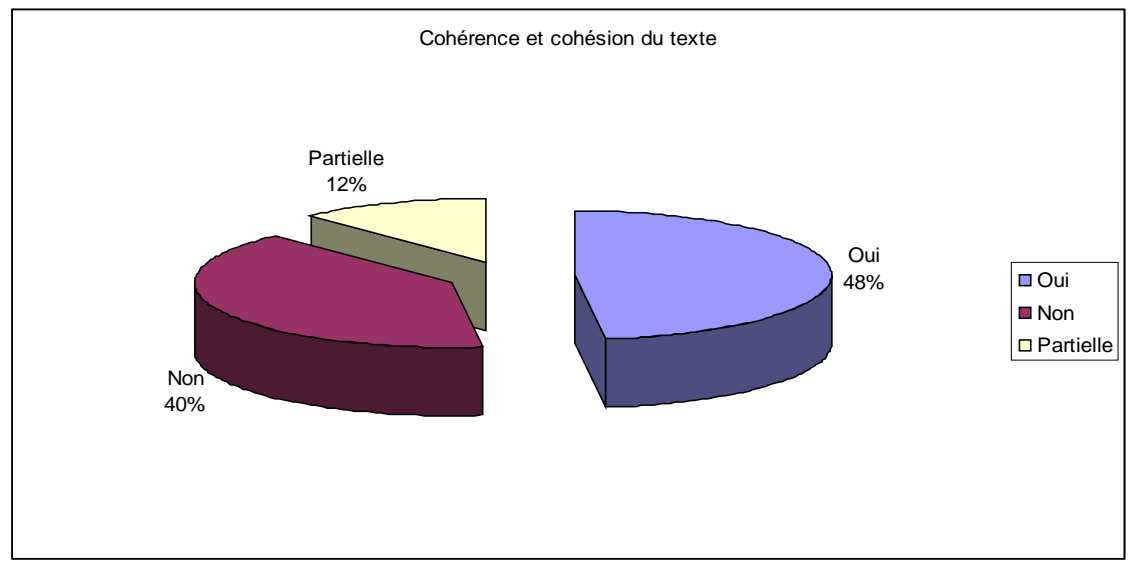

Fig. 5. Cohérence et cohésion des notes produites

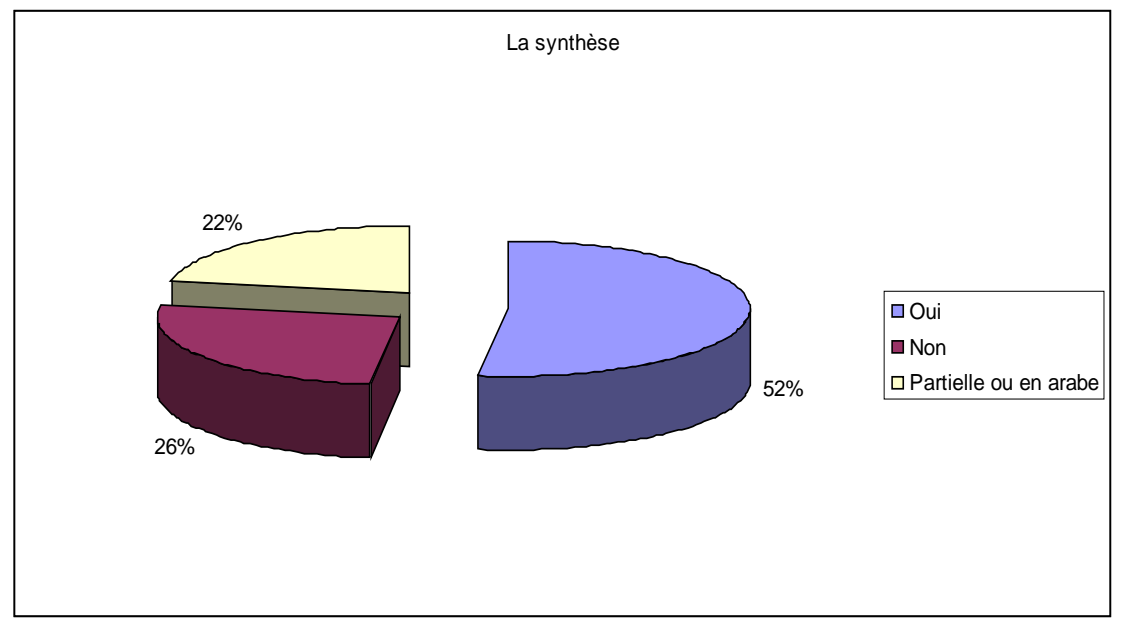

Fig. 6. Synthèse des informations recueillies 
22\% des étudiants élaborent la synthèse en langue française, 26 \% restant rédigent en langue arabe et les 52\% restants des copies ne contiennent pas de synthèse d’informations recueillies.

\section{Interprétation générale des résultats obtenus}

Les analyses ont pu relever que :

Les apprenants repèrent à peine le thème traité dans le cours magistral et recourent à la traduction dans leurs notes; $48 \%$ des copies arrivent à respecter la structure du texte avec une restitution de passages entiers du cours sans reformulation personnelle à l'exception de 7 \%. Le reste du pourcentage représente les copies où il nous a été impossible de dégager les traces du cours magistral mémorisé.

Les notes sont rédigées non pas sous forme de notes mais avec des phrases et des paragraphes entiers de l'enseignant dans lesquelles n’apparaissent ni cohérence ni cohésion.

L’absence de la langue correcte dans leurs notes est estimée à 64\% entre incorrection totale et partielle. Dans la plupart des écrits, aucune trace de sélection des parties importantes du cours (problématique, progression du cours, synthèse). Ce qui nous amène à confirmer que la difficulté de la compréhension de la langue devient un obstacle qui entrave le suivi normal du cursus chez les étudiants scientifiques car les processus mentaux (écoute, sélection, mémorisation, hiérarchisation) mis en œuvre dans ce type d’activités sont impossibles sans l'appréhension et la compréhension de la langue (Sebane, 2008).

\section{Conclusion}

Cette enquête élaborée auprès des étudiants en sciences économiques et les résultats qui en découlent représentent la continuité d'un travail menée en amont dans le cadre du projet CRASC sous le thème de l'élaboration d'un référentiel de compétences en FOU pour les étudiants de master.

En effet, une première enquête dans le cadre de ce projet auprès d'enseignants de langue et de spécialité via des questionnaires avait révélé que les enseignants des spécialités avaient conscience des lacunes des étudiants mais ne collaboraient pas avec les enseignants de langue (Mangiante et Parpette, 2004) afin de tenter de trouver des solutions. De plus, même ces enseignants de langue ne possédaient pas d'outils méthodologiques en ingénierie pédagogique et de formation pour intervenir dans ce type de situations-problèmes. Et que donc la formation des enseignants en FOS/FOU reste indispensable.

D’après les analyses des copies d'étudiants effectuées, les résultats ont démontré que les savoir-faire exigés dans une telle situation d'apprentissage n’étaient pas installés.

Acquérir toutes ces compétences dès la première année du cursus universitaire de spécialités est nécessaire et ce via un programme qui comprend plusieurs activités pédagogiques présentées d’une manière enchainée et progressive permettant le développement de la compétence vers la fin de l'apprentissage. Ce que nous tentons de faire en collectant toutes les données nécessaires pour l'élaboration d’un référentiel de compétence en contexte algérien.

Le niveau des étudiants (A1) constitue un obstacle pour faire du FOS ou du FOU. En effet, le niveau d’entrée des étudiants ne correspond pas au profil attendu pour une formation universitaire scientifique. 100\% des questionnés ont des lacunes en langue et en langue de spécialité .Ces dernières constituent un obstacle pédagogique voire un handicap freinant les objectifs universitaires assignés par l'apprenant ou par l'enseignant.

Nous allons nous pencher dans une prochaine étape de recherche de solution rapide et efficace, sur l'analyse du discours de l'enseignant afin de pouvoir cerner la démarche pédagogique, la langue utilisée et par conséquent pouvoir élaborer des programmes de formations en FOS répondant aux besoins et attentes du public concerné. 


\section{Références bibliographiques}

CAdre Commun de References pour les Langues (2000). Apprendre, enseigner, évaluer. Strasbourg : Didier.

CuQ, Jean-Pierre et GruCA, Isabelle (2005). Cours de didactique du français langue étrangère et seconde. Grenoble : Presses Universitaire.

CuQ, Jean-Pierre (2003). Dictionnaire de didactique du français langue étrangère et seconde. Paris : CLE international.

CUQ, Jean-Pierre et GRUCA, Isabelle (2002). Cours de didactique du français langue étrangère et seconde. Ed Presses Universitaires de Grenoble (PUG).

LehmanN, Denis (1993). Objectifs spécifiques en langue étrangère. Les programmes en question. Paris : Hachette. Col. F.

MANGIANTE, Jean-Marc et PARPETTE, Chantal (2004). Le français sur objectifs spécifiques : de l'analyse des besoins à l'élaboration d'un cours. Coll. F. Paris : Hachette.

QuotB, Hani (2007).Vers une didactique du français sur Objectifs Spécifiques médiée par Internet. Thèse de doctorat Université de

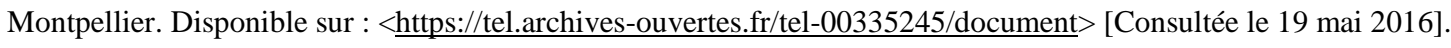

SEBANE, Mounia (2008). L'effet de deux modalités de prise d'informations sur la compréhension et la production d'un texte explicatif en FLE chez les étudiants de magistère d'économie. Thèse de doctorat es-sciences. Université de Mostaganem. Disponible sur : <http://lafef.net/bd/edaf.php?liste_theses_edaf $=\& m X=50 \& f=N V>$ [Consultée le 25 mai 2016].

Sebane, Mounia (2011). «FOS/FLE : Quel français pour les étudiants algériens des filières scientifiques ? ». Dans Actes du Forum Mondial Héraclès. Université de Perpignan Via Domitia. Gerflint, Synergies Monde, Numéro 8-2011. Tome II, 375-376.

ToLAs, Jacqueline (2004). «Le français pour les sciences : niveau intermédiaire ou avancé ». Paris : PUG. 